La revue La revue pour l'histoire du CNRS

POUR L'HISTOIRE DU CNRS

16 | 2007

L'expertise scientifique

\title{
Le risque comme objet de recherche académique
}

Claude Gilbert et Isabelle Bourdeaux

\section{(2) OpenEdition}

Journals

Édition électronique

URL : https://journals.openedition.org/histoire-cnrs/1527

DOI : 10.4000/histoire-cnrs. 1527

ISSN : 1955-2408

Éditeur

CNRS Éditions

Édition imprimée

Date de publication : 3 avril 2007

ISBN : 978-2-271-06453-0

ISSN : 1298-9800

Référence électronique

Claude Gilbert et Isabelle Bourdeaux, « Le risque comme objet de recherche académique », La revue pour l'histoire du CNRS [En ligne], 16 | 2007, mis en ligne le 26 mars 2009, consulté le 20 mai 2021. URL : http://journals.openedition.org/histoire-cnrs/1527 ; DOI : https://doi.org/10.4000/histoire-cnrs. 1527

Ce document a été généré automatiquement le 20 mai 2021.

Comité pour l'histoire du CNRS 


\title{
Le risque comme objet de recherche académique
}

\author{
Claude Gilbert et Isabelle Bourdeaux
}

La mise en œuvre au sein du CNRS

1 Le lancement des programmes CNRS «Risques collectifs et situations de crise » (1994-1996 ; 1997-1999) puis du GIS du même nom ${ }^{1}$ (2000-2005) a correspondu à une entreprise visant simultanément plusieurs objectifs :

- Inscrire la thématique des risques et des crises ${ }^{2}$ dans le champ de la recherche en sciences humaines et sociales. Contrairement à ce qui était observable dans l'univers anglo-saxon, cette thématique a tardé à apparaître en France comme un objet académique. Dans les années 1970, elle n'était traitée que par quelques chercheurs isolés, se trouvant souvent en marge de leurs institutions et se situant plutôt dans une perspective " d'ingénierie sociale ».

- Faire de cette thématique un objet «normal » pour la recherche en évitant de constituer les risques et les crises collectifs en tant que discipline. La tentation était forte, notamment dans les années 1970 et 1980, de créer un champ disciplinaire nouveau autour des « sciences du danger » (crisologie, risquologie, cindinyques, etc.).

- Faire émerger une capacité d'analyse autonome des sciences humaines et sociales qui ne se limite pas aux demandes des décideurs et experts des champs scientifiques « durs » (demandes concernant essentiellement l'analyse de la perception des risques, l'analyse de l'acceptabilité sociale des risques, des innovations technologiques à risques, etc.); et, dans le même temps, entretenir le dialogue et développer des formes de collaborations tant avec les acteurs qu'avec les chercheurs des autres sciences.

Différentes opérations de structuration et animation de la recherche

Dans le cadre des programmes CNRS, trois appels à propositions de recherche ont été lancés (1994, 1997, 1998), permettant le financement de 34 recherches (et/ou séminaires), certains d'entre eux étant co-financés avec d'autres programmes du CNRS, dans un souci d'interdisciplinarité ${ }^{3}$. Une dizaine de projets n'ont pas été financés directement par le CNRS mais ont trouvé le soutien d'institutions partenaires (ministère de l'Environnement, Inra, IRSN). Ce soutien aux recherches est également passé par des collaborations avec différents programmes du CNRS ou de ministères, via 
les chercheurs participant au conseil scientifique des programmes puis du GIS « Risques collectifs et situations de crise " ${ }^{4}$. Ainsi, de nombreux chercheurs, confirmés ou jeunes chercheurs, ont-ils pu investir différents types de problématiques, avec parallèlement des moments de confrontation et de valorisation de leurs travaux ${ }^{5}$.

Dans le cadre des programmes CNRS comme du GIS, une autre partie essentielle de l'action de structuration et d'animation de la recherche sur les risques et les crises s'est en effet effectuée à travers différents types de séminaires, ateliers et colloques, avec dans la mesure du possible la réalisation d'actes largement diffusés :

- des séminaires réguliers, organisés tant au CNRS qu'à l'École des Mines de Paris, lors desquels des chercheurs reconnus étaient invités à exposer comment, dans leurs propres champs, étaient abordées les questions des risques et des crises ou, plus précisément, comment les apports de leurs disciplines pouvaient permettre de renouveler les problématiques.

- des cycles de séminaires plus inter-disciplinaires, et associant également des acteurs opérationnels : séminaires sur le retour d'expérience, l'apprentissage et la vigilance organisationnels ; séminaire sur la prise en compte des vulnérabilités en matière de risques sismiques ; séminaire sur l'expertise en situation d'incertitude ; école thématique organisée conjointement par les départements SHS et SDU sur l'expertise dans le domaine de l'environnement...

4 Parmi ceux-ci, on peut souligner l'importance et l'intérêt de l'opération interdisciplinaire "Le risque de défaillance et son contrôle par les individus et les organisations dans les activités à hauts risques ", organisé sur trois ans (2000-2003), en partenariat avec l'ACI Cognitique du ministère de la Recherche et avec le soutien d'EDF, de la SNCF, de la RATP, de Dassault Aviation. Cette opération a permis, grâce à une série de séminaires résidentiels et des publications, un véritable travail interdisciplinaire entre chercheurs de différentes disciplines (sciences de l'ingénieur, sciences et technologies de l'information, neurosciences, sciences humaines et sociales) et représentants d'industrie, à travers l'analyse de différents types d'erreurs et défaillances à plusieurs niveaux : l'individu, le petit collectif, l'organisation, l'interorganisation. Cette opération a trouvé différents prolongements tant dans le domaine des transports (via la SNCF, le Predit 3) que dans celui des risques industriels (suite notamment à la catastrophe d'AZF à Toulouse en 2001) où différents chercheurs participent à la Fondation pour une culture de sécurité industrielle (mise en place dans le cadre des nouvelles fondations de recherche en partenariat public/privé).

Enfin, à signaler également, le rôle, dans la structuration d'une communauté, de la liste de diffusion électronique " Risques » (hébergée via l'unité « Réseaux » du CNRS) mise en place à partir de 2002 dans le cadre du GIS ${ }^{6}$. Cette liste, qui compte plus de 400 abonnés en 2006 (chercheurs, étudiants, mais aussi acteurs institutionnels du secteur privé et public) permet, via une veille scientifique, la diffusion d'informations sur des annonces de manifestations scientifiques, des annonces de publications récentes, d'appels à propositions de recherche et autres informations sur des financements possibles de recherche, et toute information de nature à concourir au développement de la recherche et à la diffusion des connaissances dans le domaine des risques et des crises. Des objectifs globalement atteints

6 Un assez grand nombre de chercheurs en sciences humaines et sociales ont investi aujourd'hui le champ de recherche des risques et des crises qui, désormais, tendent à être considérés comme des objets de recherche à part entière. Une série de thèses ont 
été soutenues ou sont en cours, explorant différents types de problématiques. Une importante littérature scientifique est désormais disponible sur des thèmes et objets diversifiés, ouvrant la voie à la poursuite d'études de cas comme à des travaux de synthèse. Des connexions, encore à développer, se sont opérées, au fil du temps, entre chercheurs relevant d'équipes et de courants de recherche différents.

7 Dès lors que les risques et les crises ont été clairement présentés comme un objet (et non comme une discipline), de très nombreuses disciplines et sous-disciplines s'en sont saisi, notamment: les sciences politiques (analyse des politiques publiques), la sociologie de l'action collective, la sociologie des organisations, la sociologie des sciences et des techniques, la sociologie du travail, l'ergonomie cognitive, les sciences juridiques, l'histoire, la géographie, etc.

8 Une capacité autonome d'analyse s'est clairement affirmée à travers l'investissement de questions telles que les conditions d'émergence des risques comme problème public, les modes de constitution des systèmes d'acteurs autour des risques, l'action publique en matière de prévention des risques, les modalités de développement et de gestion des situations de crise. Mais également sur des questions plus directement liées à des préoccupations opérationnelles telles que les conditions de mise en place des procédures de retour d'expérience, de l'expertise scientifique en situation d'incertitude, etc.

Quelques réserves toutefois

Un large bilan effectué en 2005 a cependant permis de faire apparaître certaines limites ${ }^{7}$. La recherche sur les risques et les crises, qui appelle l'interdisciplinarité (au sein des SHS mais aussi avec les autres sciences) s'inscrit encore difficilement dans un paysage qui demeure fortement marqué par le découpage disciplinaire. De façon liée, l'articulation recherche/enseignement ne s'est pas véritablement effectuée dans ce domaine, les risques et les crises restant avant tout des thématiques de recherche (ce qui n'est pas sans poser des problèmes en termes de formation, de cursus, de carrière). La recherche sur les crises demeure toujours difficile, notamment sous l'angle de l'accès aux terrains et acteurs, des problèmes de confidentialité. Différents terrains de recherche, notamment dans les entreprises à risques, dans l'univers hospitalier, restent encore insuffisamment explorés.

10 Bien qu'une dynamique interdisciplinaire ait été engagée, elle n'a cependant pas permis d'intéresser l'ensemble des disciplines relevant des SHS. Certaines, comme l'économie, l'anthropologie, la psychologie, la psycho sociologie, ainsi d'ailleurs qu'une partie de la géographie, n'ont participé que de manière marginale aux travaux des programmes/GIS «Risques collectifs et situations de crise». Par ailleurs, cette confrontation interdisciplinaire, très riche, ne va pas toujours de soi. La constitution d'un cadre d'analyse empruntant à diverses disciplines des SHS s'avère difficile même si, de fait, ce cadre a pu émerger au carrefour de certaines disciplines.

11 Enfin, l'autonomisation de la capacité d'analyse des sciences humaines et sociales a parfois rendu difficile le dialogue avec les décideurs, les experts, mais aussi avec la partie des SHS qui se cantonne à l'analyse de la perception des risques, de l'»acceptabilité ». Diverses catégories de décideurs et de chercheurs relevant des "sciences dures» ont pu être convaincues de la nécessité d'élargir le champ d'intervention des SHS, comme par exemple les physiciens, chimistes et géologues ayant participé à une réflexion interdisciplinaire autour de la question des déchets 
nucléaires ${ }^{8}$. Mais beaucoup ont une vision très classique et un peu instrumentale du rôle des SHS.

Et demain?

Comment maintenir autour de cette thématique un réseau de recherche en sciences humaines et sociales, à la fois interdisciplines et inter-organismes ? En effet, ce n'est qu'en rassemblant dans des dispositifs souples des chercheurs et des équipes dispersés dans différents laboratoires qu'il est possible de constituer une capacité de recherche significative au plan français, en mesure d'intervenir au plan européen et international. Comment articuler la thématique des risques et des crises à des thématiques plus vastes comme l'environnement ou le développement durable? Des liens évidents existent, mais les passages n'ont pas encore été effectués avec, comme difficulté, des possibilités de dilution des problématiques plus spécifiquement liées aux risques et aux crises.

Enfin, comment constituer une capacité d'analyse tirant pleinement parti des ressources propres des SHS et répondre aux demandes des décideurs et experts formulées le plus souvent en termes conventionnels? La question qui se pose est donc celle du développement d'une expertise scientifique s'appuyant fortement sur la recherche en SHS et pouvant être "entendue » par les différents décideurs et experts intervenant, à un titre ou à un autre, dans la gestion des risques.

\section{NOTES}

1.En partenariat avec le CNRS, l'Inra, l'Inserm, l'IRSN (Institut de radioprotection et de sûreté nucléaire), le ministère de l'Équipement, des transports et du logement, le ministère de l'Écologie et du développement durable, le ministère des Affaires sociales, du travail et de la solidarité.

2.Risques naturels, technologiques, biotechnologiques, sanitaires, alimentaires, environnementaux, risques associés au fonctionnement de grands réseaux (électricité, eau, télécommunications...) et crises qui leur sont associées. Ne sont pas concernées les questions de terrorisme, de violences urbaines, d'incivilités...

3.Programme "Santé et société » (axe " la sécurité sanitaire et ses enjeux sociaux ») du CNRS, Programme national de recherche sur la prévision et la prévention des risques naturels (PNRN) de l'INSU, Programme PACE (Aval du cycle électronucléaire) du CNRS. 4.Synergies par exemple avec le programme "Sciences biomédicales, santé et sociétés " du CNRS-Inserm-MiRe/DREES ; avec le programme «Évaluation et prise en compte des risques naturels et technologiques » du ministère de l'Écologie et développement durable...

5.Cf. notamment le colloque « Risques collectifs et situations de crise : bilans et perspectives » qui s'est déroulé les 7, 8 et 9 février 2001 dans l'auditorium du siège du CNRS à Paris et a fait l'objet d'un ouvrage collectif sous le titre éponyme aux éditions L'Harmattan en 2003.

6.L'animation de cette liste se poursuit actuellement dans le cadre de l'axe « Risques et crises collectifs » de la MSH-Alpes, à Grenoble. 
7. Risques, crises et incertitudes : pour une analyse critique ». Olivier Borraz, Claude Gilbert, Pierre-Benoît Joly, Cahiers GIS Risques Collectifs et Situations de Crise, $\mathrm{n}^{\circ} 3$, mars 2005, Grenoble, CNRS GIS « Risques collectifs et situations de crise ».

8. «Recherche et déchets nucléaires. Une réflexion interdisciplinaire », Cahiers Risques Collectifs et Situations de Crise, n5, février 2006, Grenoble, CNRS-MSH-Alpes.

\section{RÉSUMÉS}

Le Groupement d'intérêt scientifique (GIS) «Risques collectifs et situations de crise ", créé en juillet 2000 s'est achevé en 2005. Il visait à relayer et élargir l'action engagée par le Programme «Risques collectifs et situations de crise » du CNRS. À l'heure des bilans, les résultats sont plutôt positifs même si certaines interrogations restent sans réponse et mériteraient un prolongement des débats.

The Scientific interest network "Collective Risks and Crisis Situation", created in July 2000, ended in 2005. It aimed to take over and to widen the action opened by the Program "Risques collectifs et situations de crise" of the CNRS. For the time being, the results seem quite positive whereas a few questions are still going unanswered and would deserve an extension of the debates.

\section{AUTEURS}

\section{CLAUDE GILBERT}

Claude Gilbert est directeur de recherche au CNRS dans l'unité mixte de recherche Pacte (Politiques publiques, action politique, territoires). Il a dirigé le GIS « Risques collectifs et situations de crise » et est actuellement responsable de l'axe « Risques et crises collectifs » de la Maison des sciences de l'homme-Alpes de Grenoble.

\section{ISABELLE BOURDEAUX}

Isabelle Bourdeaux est chargée de communication au CNRS à l'unité mixte de services Maison des sciences de l'homme-Alpes de Grenoble. 